\title{
1 \\ Mechanically adaptive nanocomposites for neural interfacing
}

\author{
Jeffrey R. Capadona, Dustin J. Tyler, Christian A. Zorman, \\ Stuart J. Rowan, and Christoph Weder
}

\begin{abstract}
The recording of neural signals with microelectrodes that are implanted into the cortex of the brain is potentially useful for a range of clinical applications. However, the widespread use of such neural interfaces has so far been stifled because existing intracortical electrode systems rarely allow for consistent long-term recording of neural activity. This limitation is usually attributed to scar formation and neuron death near the surface of the implanted electrode. It has been proposed that the mechanical property mismatch between existing electrode materials and the brain tissue is a significant contributor to these events. To alleviate this problem, we utilized the architecture of the sea cucumber dermis as a blueprint to engineer a new class of mechanically adaptive materials as substrates for "smart" intracortical electrodes. We demonstrated that these originally rigid polymer nanocomposites soften considerably upon exposure to emulated physiological and in vivo conditions. The adaptive nature of these bioinspired materials makes them useful as a basis for electrodes that are sufficiently stiff to be easily implanted and subsequently soften to better match the stiffness of the brain. Initial histological evaluations suggest that mechanically adaptive neural prosthetics can more rapidly stabilize neural cell populations at the device interface than rigid systems, which bodes well for improving the functionality of intracortical devices.
\end{abstract}

\section{Introduction}

Neural prosthetic devices, which (re)connect the brain with the outside world, promise to be useful for many clinical applications..$^{1-9}$ Microelectrodes that are inserted into the brain and left to reside in the neuronal cell body layers of the cortex are the key element to this technology. ${ }^{3}$ Such electrodes can record the activity of individual or small populations of neurons for a short time after implantation, ${ }^{8,10,11}$ but it has proven difficult to record neural signals over long periods. ${ }^{3}$ This remains one of the largest hurdles in translating research successes in this field into clinical implementation and standard of care. ${ }^{3,6,12-14}$

While the dominant mechanism is still being debated, it is well established that the recording ability of intracortical microelectrodes is related to the proximity of viable neurons and the characteristics of non-neural tissue between the electrode and neurons. ${ }^{10,15}$ The most widely accepted hypothesis for electrode failure is the development of an encapsulating scar at the electrode/tissue interface. ${ }^{3,12,13,15-17}$ Insertion-related damage is practically unavoidable and causes an acute tissue response; this effect can be minimized by reducing the insertion trauma. ${ }^{18-20}$ For example, Sharp and colleagues have shown that implanting the device more slowly can reduce initial scar formation..$^{20}$ Interestingly, however, several studies showed that the chronic response is independent of the acute reaction induced by surgical trauma. ${ }^{15,19}$ Such studies have revealed two important phenomena: (1) the formation of a dense encapsulating scar, which eventually encapsulates the electrodes, regardless of the materials used; and (2) the formation of a neuron-free dead zone surrounding the implants. ${ }^{3,1521-23}$ Numerous factors are thought to contribute to scar formation, including the mechanical properties mismatch between stiff electrodes and the soft cortical tissue, ${ }^{24-26}$ and a localized neurotoxic environment created by the presence of a non-removable foreign object. ${ }^{22-24}$ The aim of this article is to summarize the different approaches to stabilize the neural electrode/brain interface by focusing on the mechanical mismatch of the implant and tissue, and, as an example, highlight recent work on mechanically adaptive nanocomposites for neural interfacing.

Jeffrey R. Capadona, Department of Biomedical Engineering, Case Western Reserve University, Cleveland, OH 44106, USA; jrc35@case.edu Dustin J. Tyler, Department of Biomedical Engineering, Case Western Reserve University, Cleveland, OH 44106, USA; dustin.tyler@case.edu

Christian A. Zorman, Department of Electrical Engineering and Computer Science, Case Western Reserve University, Cleveland, OH 44106, USA; Christian.Zorman@case.edu

Stuart J. Rowan, Department of Macromolecular Science and Engineering, Case Western Reserve University, Cleveland, OH 44106, USA; stuart.rowan@case.edu

Christoph Weder, Adolphe Merkle Institute, University of Fribourg, Switzerland; christoph.weder@unifr.ch

DOI: $10.1557 / \mathrm{mrs} .2012 .97$ 


\section{Strategies for stabilizing the neural electrode/brain interface}

Most experimental microelectrodes are made from metals, silicon, and/or ceramics (i.e., materials that are much stiffer [Young's modulus, $E \sim 200 \mathrm{GPa}]$ than cortical brain tissue [E 0.1-6 kPa]). These rigid materials generally enable facile electrode insertion and have provided many of the major advances in the field. . $^{3,5,27}$ However, once implanted, they exert forces on the surrounding tissue, since pulsing, respiration-related, and everyday motions cause movements of the brain relative to the device. ${ }^{28}$ The resulting shear forces scale with the stiffness difference between tissue and implant. ${ }^{29}$ For example, electrodes placed into cortical tissue without anchoring to the skull (referred to as "un-tethered") exhibit smaller differential motions than identical electrodes that are anchored to the skull (referred to as "tethered") and cause less inflammatory-mediated scarring. ${ }^{30}$ Mechanical modeling predicts that stresses induced in the surrounding tissue are reduced if the electrode's stiffness is lowered, ${ }^{29}$ and better tissue integration will minimize strain. ${ }^{25}$ Therefore, the effects of the mechanical mismatch are thought to play a significant role in the cell-mediated inflammatory response affecting the microelectrode/cortical tissue interface. .,16,21,31

Several strategies are being investigated to improve microelectrode biocompatibility and to minimize inflammatory responses, ${ }^{6,21}$ typically by promoting a more intimate interface between the neurons and the electrode surface. Several therapeutic approaches have demonstrated temporal attenuation of certain aspects of foreign body encapsulation and/or neuronal dieback at the device interface, ${ }^{17,32-39}$ but a clinically viable approach for long-term recording without the risk of significant side effects has yet to be developed. Several groups investigated the effects of mechanical mismatch between microelectrodes and cortical tissue by fabricating microelectrodes and, in some cases, auxiliary coatings from a variety of "off-the-shelf" polymeric materials..$^{40-46}$ In general, these materials have yet to demonstrate success in attenuating scar formation or improving neural recordings, perhaps because most of the materials employed had Young's moduli that were still $10^{6}$ times higher than that of the brain. ${ }^{42,43,47-49}$ Implanting compliant electrodes is difficult, ${ }^{42,50-52}$ because soft devices buckle during (attempted) insertion into cortical tissue unless special measures are taken..$^{50}$ Such special measures are often only applicable to single shank electrode systems and are difficult, if not impossible, to apply to arrayed electrode devices. Mechanical modeling reveals that a Young's modulus of several GPa is required to allow for unassisted insertion of the electrode with a cross-sectional area of around a thousand $\mu \mathrm{m}^{2}$ into cortical brain tissue. On this basis, significant efforts were made to develop electrodes based on semi-flexible polymer substrates and "hybrids" between rigid silicon cores and soft hydrogel coatings (for a review, see Reference 53).

\section{Comparison of mechanically adaptive nanocomposites to other electrode materials}

The concept of using mechanically adaptive nanocomposites as the basis for cortical electrodes potentially offers some advantages over the current generation of electrode materials. Perhaps most importantly, the soft modulus after implantation (ca. $10 \mathrm{MPa}$ ) is significantly lower than that of silicon or tungsten $(\sim 100 \mathrm{GPa})^{54}$ or even that of other flexible polymers used in neural interfacing, such as polyimide, ${ }^{40}$ poly(benzocyclobutene), ${ }^{47}$ or poly( $p$-xylylenes) (parylene) $(\sim 2-3 \mathrm{GPa}) .^{42,55}$ Furthermore, electrodes based on such flexible polymers require temporary stiffening to allow for implantation, something that is not required by an appropriate mechanically adaptable material (vide infra). Nonetheless, a number of innovative approaches have been explored to achieve temporary stiffening of a polymer-based electrode. For example, the coating of a polymer device with biodegradable rigid polymers such as poly(lactic-co-glycolic acid) (PLGA), deposition of a thin layer of silicon, or the use of a "carrier" to insert the electrode. ${ }^{50,52,56}$ More recently, Shain and co-workers reported a biodegradable terpolymer coating system with a modulus of around $400 \mathrm{MPa}$, which required a larger than normal cross-sectional area of the electrode to allow for reliable implantation. ${ }^{57}$ While each of these approaches has been demonstrated to be useful for the insertion of simple single-shank electrodes, challenges still exist if they are to be applied to multi-shank probes with an integrated lead cable. Furthermore, the use of a "carrier" system (i.e., a rigid insertion device around the electrode, which is retracted after insertion) to deliver the microelectrode also increases the volume of damaged tissue. Several studies have suggested that the damage caused during insertion of the electrodes will eventually heal, and neurons will repopulate the damaged tissue (vide infra). However, to date, no such studies have been reported on "carrier" models, and it is still unclear if such carriers will compound the wound healing response with the addition of a foreign body reaction. Finally, the use of a degradable coating adds an additional level of complexity in evaluating the bio-response, as one must also consider how the degradation products may affect the inflammatory response in the surrounding tissue.

Another interesting approach, pioneered by Martin and co-workers, relies on the coating of rigid electrodes with hydrogels that can reach $E$ valves lower than the current generation of mechanically adaptive nanocomposites..$^{58-63}$ The hydrogel is dehydrated prior to implantation to render it stiff and prevent it from being stripped off the electrode during insertion. Once inserted, the coating absorbs large amounts of water from the tissue, causing it to swell and form a very soft hydrogel. It is yet unclear if the hydrogels, which serve as a mechanical "buffer," are capable of masking the mechanical strain on the tissue, or if the underlying bulk stiffness will play a significant role in host response. The extensive swelling of the hydrogel coating increases the volume occupied by the electrode after insertion and "pushes" the neurons away from the actual electrical contact. To address this point, recent approaches to bridge the gap between the electrode and the tissue involve the grafting of conductive polymers such as polypyrrole or poly(3,4-ethylenedioxythiophene) (PEDOT), ${ }^{64,65}$ or bioactive peptides to promote neuronal ingrowth onto the surface of the 
electrodes. ${ }^{66,67}$ Immunohistological results of in vivo testing of the hydrogel electrode system have yet to be reported, but recording studies suggest improved signal quality in short-term animal studies. ${ }^{58,59}$

\section{Innovation through bioinspiration}

We set out with the goal of creating a microelectrode that would be sufficiently stiff to be easily inserted into the brain, but would subsequently soften upon implantation to better match the stiffness of the cortex, thereby minimizing forces exerted on the tissue and attenuating inflammation. ${ }^{68}$ To this end, we have developed a new class of bioinspired, chemoresponsive, mechanically adaptive polymer nanocomposites that can controllably and selectively be switched between stiff and compliant states. ${ }^{68-74}$ The design of these materials is based on the architecture and functionality of the sea cucumber dermis. $^{75-77}$ These invertebrates can rapidly and reversibly switch the stiffness of their skin (Figure 1a-b) ${ }^{78-81}$ This response is achieved through a nanocomposite design that relies on rigid collagen fibers, which reinforce a soft matrix. The stiffness of the tissue is regulated by controlling the interactions, and thereby stress transfer, between adjacent collagen fibrils. Our artificial nanocomposites mimic the sea cucumber's general design and consist of a soft polymer matrix that is reinforced with rigid nanofibers (Figure 1c). The magnitude of the reinforcement depends mainly on the modulus of the nanofibers, their aspect ratio (ratio of length to width), and concentration in the matrix, as well as their interactions between each other and with the polymer matrix. In the "on state," the
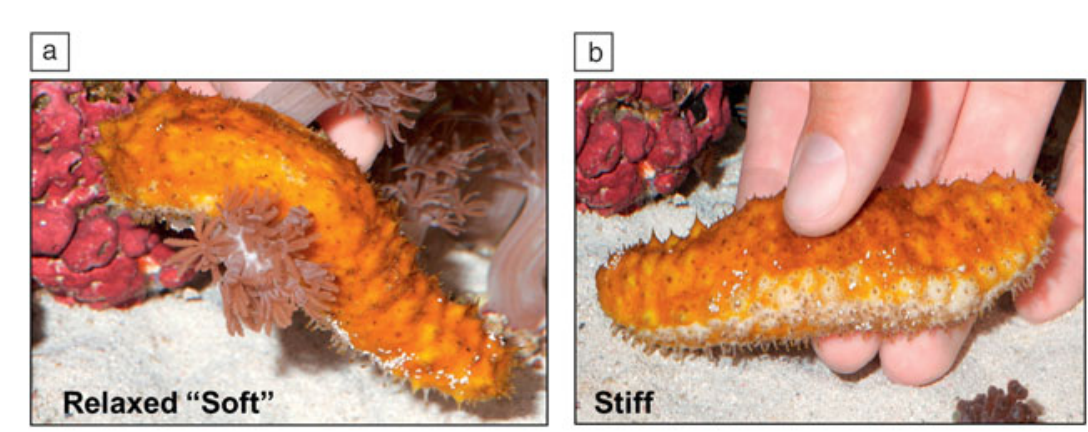

C

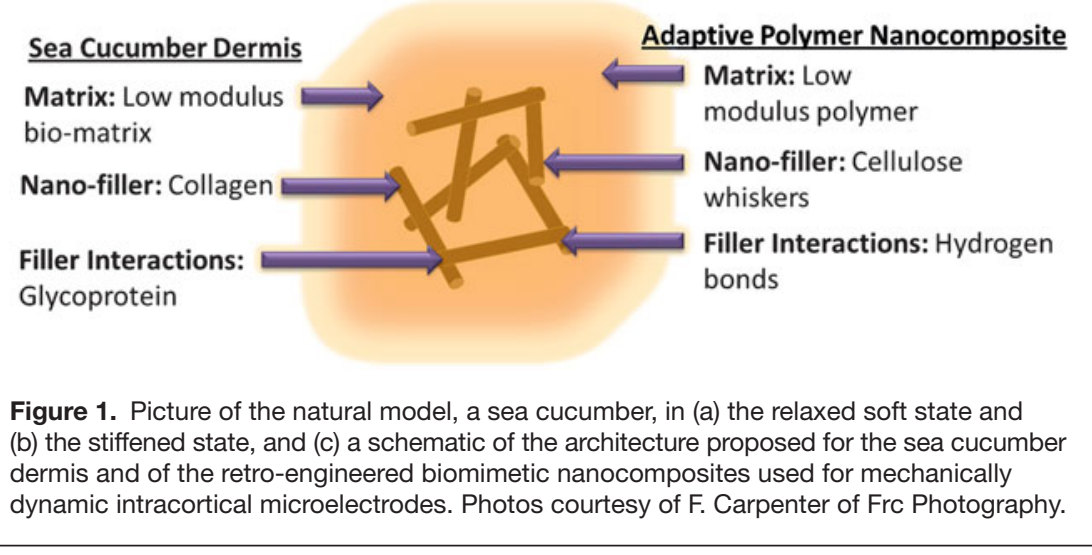

nanofibers strongly interact with each other so that they form a load-bearing percolating network, leading to a high overall stiffness. Upon introduction of a chemical switch that turns off the interactions between the nanofibers, the load-bearing network is disassembled, and the material's stiffness is reduced. ${ }^{68,72,82}$

\section{Multiple generations of artificial, mechanically adaptive nanocomposites}

To achieve the desired mechanical switching in nanofibercontaining nanocomposites, it is critical that the filler particles form a rigid reinforcing network within the polymer matrix, and their interactions can be tuned through a stimulus in a controlled manner. ${ }^{68,71,72,83}$ We chose to employ highly crystalline, bio-based cellulose nanofibers (also referred to as cellulose nanocrystals, nanowhiskers, or simply whiskers), ${ }^{83}$ which, depending on the source, display a Young's modulus of $100-150 \mathrm{GPa}^{84,85}$ an ultimate tensile strength of up to $10 \mathrm{GPa}$, and an aspect ratio of up to $100 .{ }^{86}$ Due to the abundance of surface hydroxyl groups, cellulose whiskers strongly interact with each other through hydrogen bonding, but exposure to water efficiently reduces whisker-whisker interactions on account of competitive hydrogen bonding; this feature was exploited to create water-responsive, mechanically adaptive materials. Nanocomposites, in which the cellulose whiskers form a percolating network in a polymer matrix, can be fabricated by combining the components in a hydrogen-bond-forming solvent so that the whisker-whisker interactions are "switched off." Upon film casting and evaporation of the solvent, whisker interactions are switched on, and the whiskers assemble into a percolating network within the polymer matrix.

In our first generation of mechanically adaptive nanocomposites, cellulose whiskers isolated through acid hydrolysis of the mantles from tunicates - sessile sea creatures - were employed as the responsive filler. ${ }^{68}$ These tunicate nanowhiskers (TNWs) feature a diameter of $\sim 20 \mathrm{~nm}$, an aspect ratio of $\sim 70-100$, and an on-axis tensile storage modulus $E^{\prime}$ of $120-150 \mathrm{GPa}$. Their high aspect ratio and stiffness make TNWs a better filler than cellulose whiskers from other sources, but these other sources, including wood, hemp, flax, jute, ramie, bacteria, and cotton, are often technologically more viable (vide infra). TNWs were integrated into a rubbery ethylene oxideepichlorohydrin (EO-EPI) copolymer matrix, ${ }^{68,70,87}$ which in its neat form displays a low tensile storage modulus $\left(E^{\prime}=0.3-3 \mathrm{MPa}\right.$, depending on the comonomer ratio) and can be swelled slightly with water. The incorporation of TNWs into the EO-EPI matrix causes a dramatic stiffness increase. ${ }^{70} E^{\prime}$ increased with increasing TNW content from $1.3 \mathrm{MPa}$ for the neat polymer to $800 \mathrm{MPa}$ at a TNW content of $17 \% \mathrm{v} / \mathrm{v}$.

As designed, the nanocomposites exhibit a significant stiffness reduction upon exposure 
to water (Figure 2a). For example, $E^{\prime}$ dropped from 800 to $20 \mathrm{MPa}$ for the material comprising $17 \% \mathrm{v} / \mathrm{v}$ TNWs. This response was completely reversible; when the materials were dried, the original properties were restored. ${ }^{68,70}$ The mechanical properties of these EO-EPI/TNW nanocomposites (and all others discussed here) are well described by two mechanical models: the percolation model for the dry state and the Halpin-Kardos model for the wet state (Figure 2a). These models, which are discussed in detail elsewhere, ${ }^{68,88,89}$ can be used to predict the mechanical on-off contrast of the adaptive
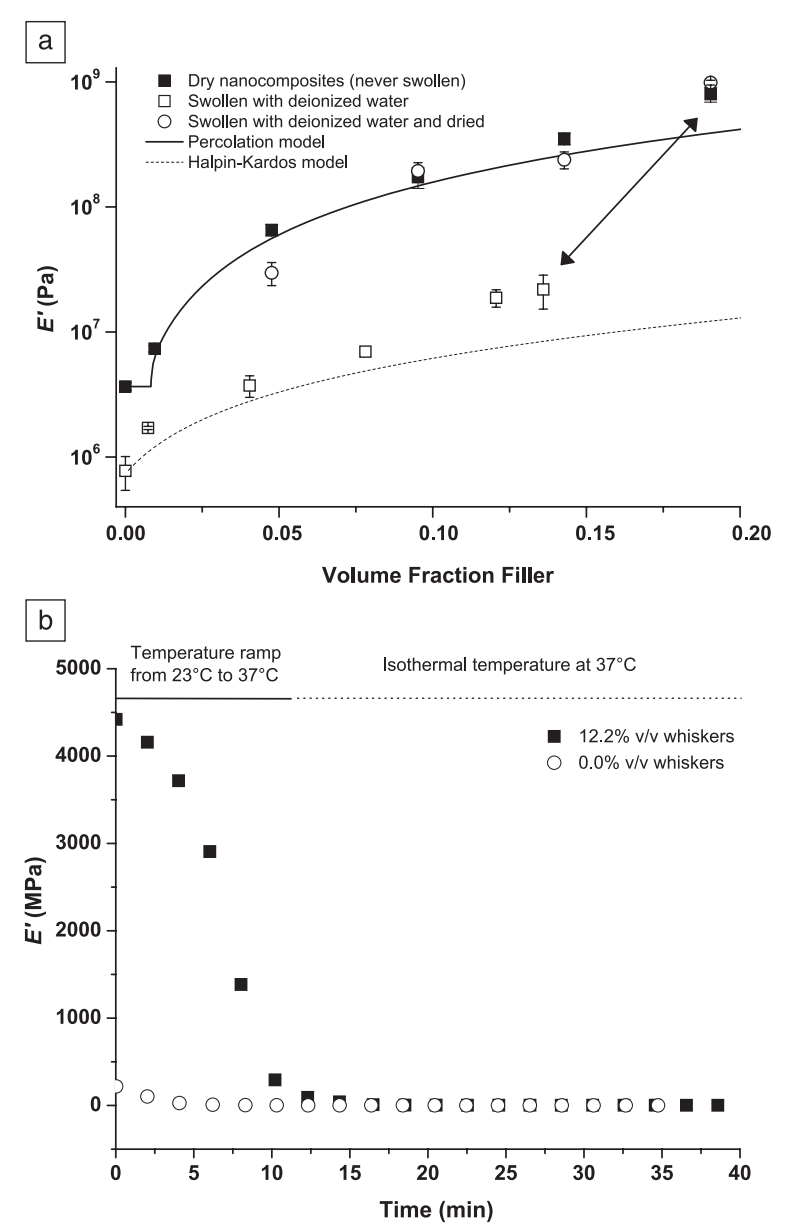

Figure 2. (a) Tensile storage moduli $E^{\prime}$ of ethylene oxideepichlorohydrin (EO-EPI)/tunicate whisker nanocomposites as a function of volume fraction of whiskers. The nanocomposites were conditioned by either drying in vacuum, equilibrium swelling in deionized water, or swelling to saturation in deionized water followed by re-drying in vacuum. The lines represent values predicted by the percolation (solid) and Halpin-Kardos (dotted) models. The arrow indicates changes in modulus and volume fraction of whiskers resulting from aqueous swelling of one selected sample $(19 \% \mathrm{v} / \mathrm{v}$ tunicate whiskers). (b) Time-dependent modulus decrease of neat poly(vinyl acetate) (PVAc) and a $12.2 \%$ v/v PVAc/tunicate whisker nanocomposite upon immersion into artificial cerebrospinal fluid (ACSF) and increasing the temperature from $23^{\circ} \mathrm{C}$ to $37^{\circ} \mathrm{C}$. Lines represent time required for temperature to increase from $23^{\circ} \mathrm{C}$ to $37^{\circ} \mathrm{C}$ and isothermal control at $37^{\circ} \mathrm{C}$. Reprinted with permission from Reference 68. (02008, AAAS. nanocomposites as a function of composition. Quantitative insights into the stress-transfer mechanisms that determine the films stiffness were gained by Raman spectroscopy. ${ }^{90}$ A diagnostic Raman band, associated with the $\mathrm{C}-\mathrm{O}$ ring stretching of the cellulose backbone, was used to quantify the local orientation of and the level of stress experienced by the cellulose whiskers. It was shown that the extent of stress-transfer is influenced by local orientation and connectivity of the cellulose whiskers; these features are governed by the processing conditions used to fabricate the materials. ${ }^{90,91}$

Unfortunately, the stiffness of EO-EPI/TNW nanocomposites ( $E^{\prime}$ of up to $800 \mathrm{MPa}$ ) is too low to allow for the fabrication of electrodes that can be inserted into the brain through the outermost protective membrane on the surface of the brain, the pia mater. The next generation of mechanically adaptive nanocomposites was therefore designed to exploit two complementary switching mechanisms. Poly(vinyl acetate) (PVAc), an amorphous polymer with a glass transition temperature $\left(T_{\mathrm{g}}\right)$ around $42^{\circ} \mathrm{C}$, was employed as the matrix and cellulose whiskers isolated from tunicates as the filler. Upon exposure to physiological conditions, the materials undergo a phase transition (water plasticizes the material and lowers $T_{\mathrm{g}}$ to below physiological temperature), and the whisker network is disassembled. This design allowed us to create adaptive PVAc/ TNW nanocomposites, which exhibit a mechanical contrast of three orders of magnitude between the dry state at room temperature $\left(E^{\prime}=5.1 \mathrm{GPa}\right.$ for a nanocomposite with $16.5 \%$ v/v TNWs) and the water-swollen state at $37^{\circ} \mathrm{C}(\sim 12 \mathrm{MPa}) .^{69,71}$ Figure 2b, which shows the stiffness of a thin film of a PVAc/ TNW nanocomposite with $12.2 \% \mathrm{v} / \mathrm{v}$ TNWs upon introduction into artificial cerebrospinal fluid (ACSF) at $37^{\circ} \mathrm{C}$, reveals
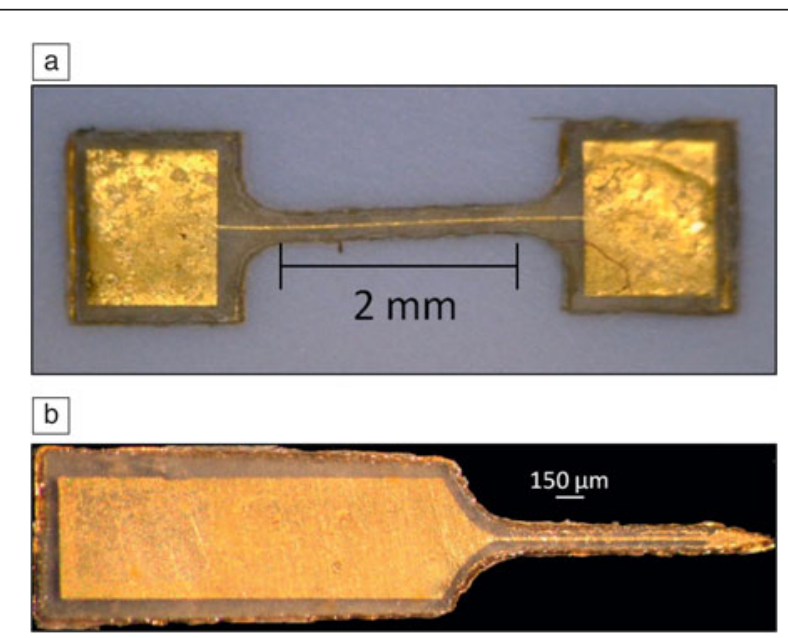

Figure 3. (a) Micromachined dogbone structure with lithographically defined Ti/Au pads and trace for evaluating mechanical properties; (b) laser-micromachined cortical probe with a lithographically defined Ti/Au electrode, interconnects, and parylene capping layers (cf. Figure 4 , entry 10). In both cases, a $12.2 \% \mathrm{v} / \mathrm{v}$ poly(vinyl acetate) (PVAc)/tunicate whisker nanocomposite was used as the mechanically adaptive substrate (thickness $60 \mu \mathrm{m}$ ). Reprinted with permission from Reference 95. (2011, IOP Publishing. 


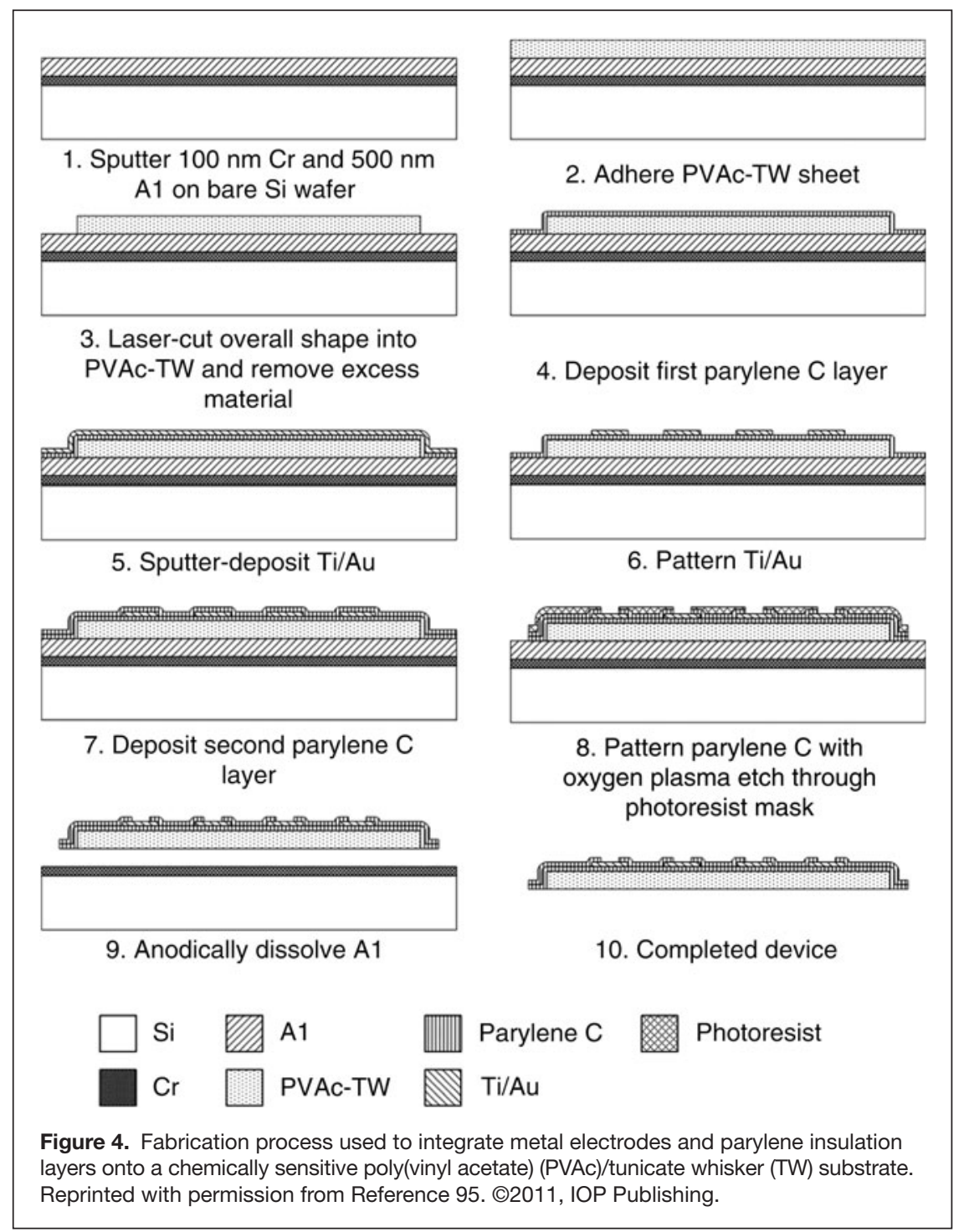

nanocomposites. Gratifyingly, PVAc/CNW nanocomposites show dynamic mechanical properties that are close to those of PVAc/ TNW nanocomposites, but their water take-up is greatly reduced $(28 \% \mathrm{w} / \mathrm{w}$ for a material with $16.5 \% \mathrm{v} / \mathrm{v} \mathrm{CNWs}$ at $\left.37^{\circ} \mathrm{C}\right) .^{72}$

Mechanically adaptive CNW nanocomposites with a rubbery polyurethane matrix were also explored. ${ }^{93}$ These materials are characterized by high toughness, exhibit water-responsive, mechanically adaptive properties, and also display shape-memory behavior. In the waterswollen, softened state, tensile deformation of the nanocomposites causes uniaxial orientation of the CNWs, which is retained upon drying the materials. The resulting oriented network fixates a temporary shape, which due to the elastic nature of the polyurethane matrix relaxes to the original shape upon wetting. Thus, this latest generation of water-triggered mechanically adaptive materials not only permits the fabrication of cortical electrodes, which soften after implantation, but also allows one to pre-program a shape change to correspond to movements that could simplify electrode placement in the tissue, or even facilitate a secondary position of the electrode, after the foreign body response has stabilized.

\section{Implant fabrication and in vivo deployment}

With this family of mechanically adaptive, physiologically responsive materials in hand, model microprobes consisting of neat PVAc only (as reference) and 12.2\% v/v PVAc/TNW nanocomposites were created (Figure 3), with the goal of implanting them into a living rodent to study the inflammatory response..$^{94,95}$

that most of the softening occurs within minutes (i.e., over a timeframe that appears to be very useful for the electrodes to be placed into neural tissue).

One perceived drawback of the PVAc/TNW nanocomposites is their rather significant water or ACSF take-up $(\sim 70-90 \% \mathrm{w} / \mathrm{w}$ for materials with $16.5 \% \mathrm{v} / \mathrm{v}$ TNWs at $\left.37^{\circ} \mathrm{C}\right)$. An excessive level of swelling can lead to delamination of the multilayer electrode structure and increases the trauma. This problem was alleviated by introducing cellulose nanowhiskers (CNWs) isolated from cotton into PVAc. The CNWs have a diameter of 10-20 nm, a length of 100-250 nm, an average aspect ratio of $\sim 10$ (which is much lower than the aspect ratio of TNWs [70-150]), and a tensile storage modulus of $\sim 105 \mathrm{GPa}^{69,92}$ The CNWs also feature a lower density of surface sulfate groups $(\sim 31 \mathrm{mmol} / \mathrm{kg})$ than TNWs $(\sim 85 \mathrm{mmol} / \mathrm{kg})$. These anionic sites, introduced during hydrolysis of the cellulose pulp with sulfuric acid, aid in their dispersion, but they are also thought to be responsible for the hygroscopic nature of the TNW-based
This first required the development of microfabrication processes compatible with the properties (rheology, chemical, and thermal stability) of these nanocomposites to allow for the integration of electrode materials and additional insulation layers. ${ }^{95}$ Based on the process shown in Figure 4, micromachined probes $(50-100 \mu \mathrm{m}$ thick) were fabricated that incorporated parylene as an insulating/moisture barrier layer $(1 \mu \mathrm{m})$ and Ti/Au contacts $(50 \mathrm{~nm} \mathrm{Ti}, 200 \mathrm{~nm} \mathrm{Au})$.

The characterization of probes manufactured by this approach revealed that the parylene and gold layers, in spite of their intrinsic stiffness, only marginally contributed to the overall stiffness, due to their comparably small thickness. Gratifyingly, these electrodes exhibit impedances $(95.0 \pm 4.8 \mathrm{k} \Omega)$ that are comparable to those of $\mathrm{Au}$ electrodes $(130 \mathrm{k} \Omega)$ on conventional substrates. Interestingly, the swelling of the nanocomposite-based electrodes was highly anisotropic, perhaps on account of processing-related anisotropy ${ }^{83}$ the expansion in the thickness dimension exceeded that of the in-plane 


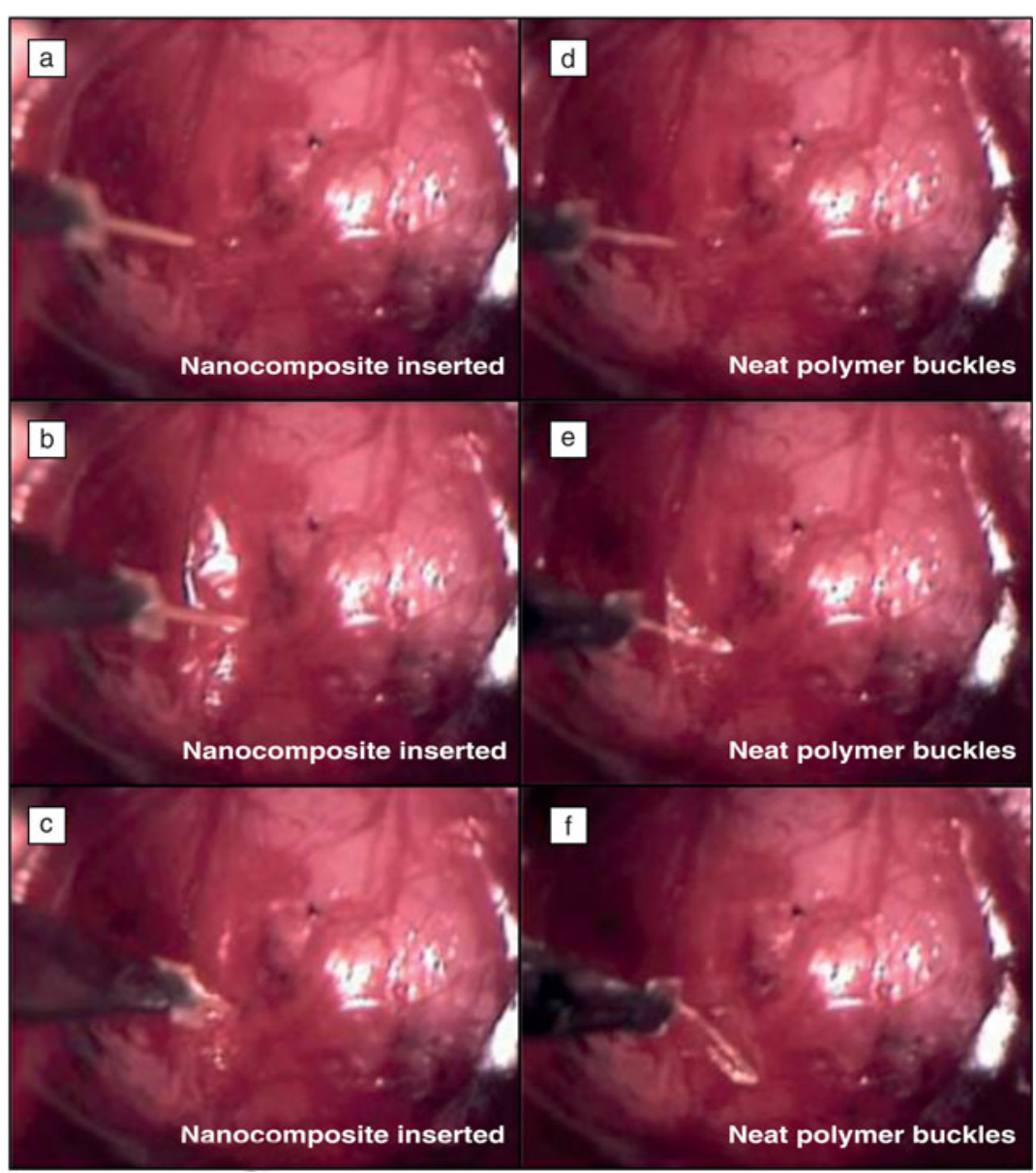

Figure 5. Snapshots of a movie that show insertion attempts of microprobes consisting of an adaptive $12.2 \% \mathrm{v} / \mathrm{v}$ poly(vinyl acetate) (PVAc)/tunicate whisker nanocomposite (NC) and (as reference) the neat PVAc polymer into the brain of a rat. (a) Before initial insertion, the NC is about one millimeter above the brain's surface. (b) Upon movement, the NC indents and then penetrates the pia and cortex. (c) After the completion of movement, the NC is implanted in the brain, and the indentation is relaxed. (d) Before initial insertion, the neat polymer is about one millimeter above the brain's surface. (e) Upon movement, the neat polymer indents the pia, but quickly buckles. (f) After the completion of movement, the neat polymer is completely buckled. Reprinted with permission from Reference 94. (C2011, IOP Publishing. To review the movie, please visit http://dx.doi.org/10.1557/mrs.2012.97.

neat PVAc buckled under lower loads $(\sim 7 \mathrm{mN})$ before they could be inserted into the cortical tissue.

We demonstrated the material's ability to morph while in the rat cortex by implanting samples into the rat cortex for up to 30 minutes, removing them, and quickly placing them in a custom-built microtensile testing setup. ${ }^{95}$ Indeed, such ex vivo samples displayed very similar mechanical morphing profiles as those measured in vitro on a dynamic mechanical analyzer (DMA, Figure 6). Taken collectively, this work demonstrated the first stand-alone material that was capable of facile insertion into the brain, while rapidly softening to more closely match the mechanical properties of its surroundings.

The new materials allowed us to conduct an in vivo study that probed the neural inflammatory response as a function of mechanical characteristics of the probe. To accomplish this, microprobes of the mechanically dynamic $12.2 \% \mathrm{v} / \mathrm{v}$ PVAc/TNW nanocomposite were allowed to remain implanted into the rat cortex for either four or eight weeks (Figure 7). ${ }^{96}$ The inflammatory and neurodegenerative responses to these materials were compared to contralateral implants of much stiffer (and not adaptive) tungsten microwires of similar dimensions. The latter were coated with a thin layer of neat PVAc to provide matched surface chemistry and roughness. Both implants were tethered to normalize the effects of strain on the tissue, which is relevant for a recording electrode model.

Fluorescent immunohistochemistry labeling was used to examine neurons, as well as inflammatory cells. The neuronal nuclei density within $100 \mu \mathrm{m}$ of the mechanically adaptive device at four weeks post-implantation was significantly larger than that of the stiff wire control. At eight weeks post-implantation, the neuronal nuclei

direction by a factor of $\sim 10$. This feature explains, at least in part, the absence of discernible deformation and the nonexistence of delamination between the nanocomposite and parylene in the probes when deployed in deionized water. In the case of dog bone structures, used exclusively for mechanical testing (thickness of ca. $50 \mu \mathrm{m}$ ), the Young's modulus decreased from ca. 3400 to ca. $20 \mathrm{MPa}$ within only 300 seconds and displayed essentially the same mechanical contrast as the nanocomposites alone.

Figure 5 shows that microprobes of the $12.2 \% \mathrm{v} / \mathrm{v} \mathrm{PVAc/}$ TNW nanocomposite (without electrical insulation or contact layers), with a cross-sectional area of $6625 \mu \mathrm{m}^{2}$, and an angular tip could readily be inserted through the pia mater into the cerebral cortex of a rat, whereas reference probes consisting of density around the nanocomposite was maintained, but the density around the stiff control recovered to match that of the nanocomposite. While the inflammatory-mediated encapsulation of the compliant nanocomposite was less vigorous than to the stiffer wire, as quantified through detection of relevant cells via immunohistochemistry labeling, the mechanically associated cells and proteins appeared to be the most dominant modulators of the response to the compliant nanocomposite. This initial study did not involve any end points beyond a time frame of eight weeks. However, evidence in other labs, as well as our own, suggests that neurodegeneration may increase from 8 to 16 weeks post-implantation. ${ }^{12}$ This is consistent with neurotrauma literature demonstrating a biphasic response of the nervous system immune cells, or microglia, resulting in 




Figure 6. The Young's modulus of the nanocomposite material (12.2\% v/v poly(vinyl acetate)/tunicate whisker) was measured for bulk films with a dynamic mechanical analyzer (DMA) (open squares; bulk materials ${ }^{73}$ ) and microprobes with a micro tensile tester (open circles; explanted microprobes) to reflect the mechanical morphing from stiff to compliant. DMA samples were exposed in situ (i.e., in the DMA setup) to artificial cerebrospinal fluid (ACSF) that was preheated to $37^{\circ} \mathrm{C}, 7^{73}$ while microprobes were implanted into a rat cortex and explanted for mechanical testing. The $x$-axis indicates the time of exposure to either ACSF or implantation into the rat cortex, respectively. Reprinted with permission from Reference 94. (02011, IOP Publishing.

a secondary event of neurodegeneration. ${ }^{97}$ While our nondynamic, stiffer controls follow the trend of scar maturation, the dynamic nanocomposites display unique stabilization across both time points analyzed. Therefore, it appears important to probe additional time points past the eight-week mark.

\section{Summary and future directions}

Arguably, the most accepted hypothesis for electrode failure relates to the development of the encapsulating scar at the electrode/tissue interface. A growing belief is that the mechanical mismatch between current electrode materials and the cortical tissue mediates scar formation and subsequent neurodegeneration through a variety of potential mechanisms. This has led to a paradigm shift to the development of new electrodes made from softer polymeric materials and has resulted in a number of recently introduced approaches, such as the use of mechanically adaptive polymer nanocomposites or swellable hydrogels as substrates for the electrode. For example, intracortical implants based on the dynamic nanocomposite materials have been shown to be sufficiently stiff to be easily implanted into the brain and subsequently soften to better match the stiffness of the cortex. Initial histological evaluations suggest that mechanically adaptive intracortical neural prosthetics can more rapidly stabilize neural cell populations at the interface than rigid systems, which bodes well for improving the functionality of intracortical devices. However, there are still many open issues that need to be addressed. First of all, the tissue response to such adaptive materials after longer implantation times than previously studied (up to eight weeks) must be explored. Initial studies have suggested a significant advantage with the softer materials, which fluctuated with the
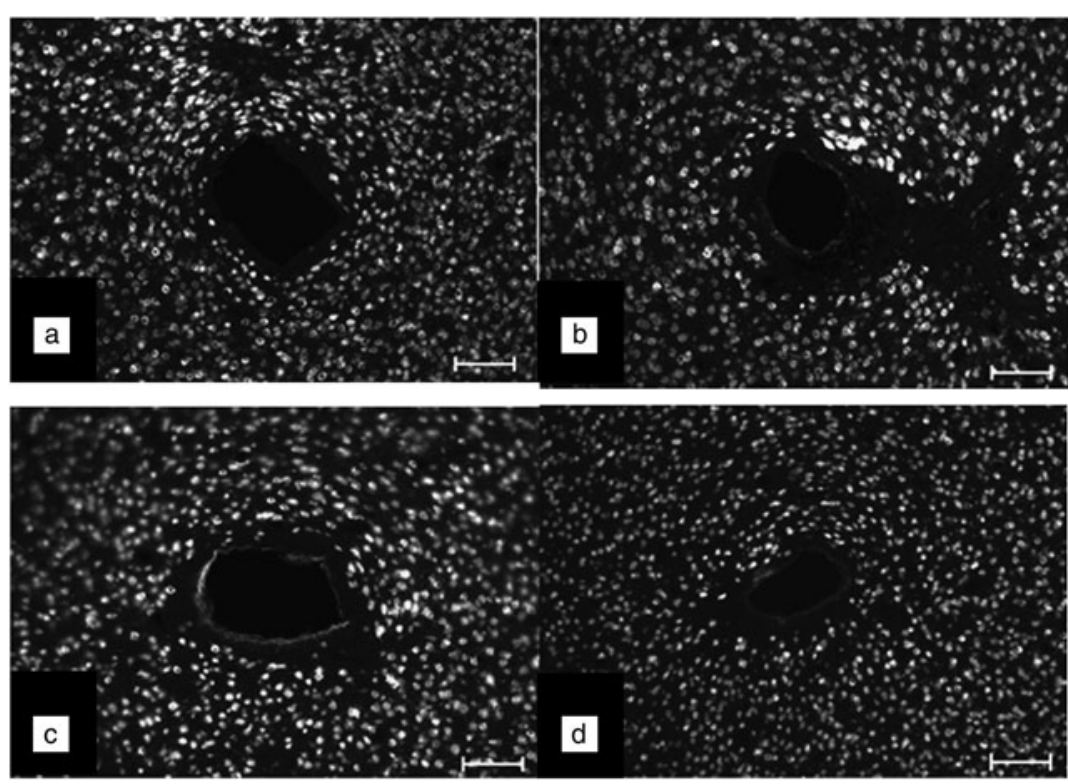

Figure 7. In order to assess the proximity of neurons to the electrode materials that were implanted into rats, we utilized standard biochemical markers for the nucleus of neurons (neuronal nuclei or NeuN) and imaged the tissue sections with a fluorescent microscope. Representative images of NeuN for tissue that had electrode materials implanted for either four $(a, b)$ or eight $(c, d)$ weeks are shown for both nanocomposite (NC) $(a, c)$ and wire (b, d) implants. Each "white" circle represents a neuron. More neurons found close to the hole created by the implant indicates less neuron death at the device/tissue interface. Scale bars are $100 \mu \mathrm{m}$. Reprinted with permission from Reference 96. (02011, IOP Publishing. maturation of the foreign body response. This suggests the potential utility of combinatorial approaches that explore both dynamic materials and short-term therapeutic measures.

Additionally, if the results confirm that softer implants are indeed better than more rigid devices, as the initial data suggest, then the question becomes what the optimal mechanical properties for cortical electrodes really are-in other words, how soft do they need to be? And is it the surface that matters or the device as a whole? This leads to the question, "What are the main biological processes that are triggered by the mechanical mismatch?" It appears that the answers to these questions are relevant to the field at large, as approaches other than the one discussed here exist to take advantage of the paradigm "softer is better." It is noted that so far, little has been done to create actual electrodes on the basis of mechanically adaptive substrates, and it is clear that such devices should be engineered and tested as soon as possible. In order for such devices to be routinely clinically viable, significant efforts to better understand the molecular and cellular events that lead to neurodegeneration at the 
device-tissue interface are needed to promote the development of materials-based and therapeutic strategies to improve device integration and stabilize neural recordings.

\section{Acknowledgments}

This work was supported by grant number R21-NS053798 from the National Institute of Neurological Disorders and Stroke (D.J.T.). Additional support was from the Department of Veterans Affairs Medical Center, specifically grant numbers: C3819C, F4827H, and B6344W (J.R.C.) and NSF grants CBET-0828155 (S.J.R./C.W.) and ECS-0621984 (C.Z.). C.W. gratefully acknowledges financial support from the Swiss National Science Foundation (NRP 62: Smart Materials, Nr. 406240_126046) and the Adolphe Merkle Foundation. Finally, some parts of this work were supported by the Department of Biomedical Engineering at Case Western Reserve University through both laboratory startup funds (J.R.C.), as well as a Medtronic Graduate Fellowship.

\section{References}

1. L.R. Hochberg, M.D. Serruya, G.M. Friehs, J.A. Mukand, M. Saleh, A.H. Caplan, A. Branner, D. Chen, R.D. Penn, J.P. Donoghue, Nature 442, 164 (2006). 2. M. Velliste, S. Perel, M.C. Spalding, A.S. Whitford, A.B. Schwartz, Nature 453, 1098 (2008)

3. M.P. Ward, P. Rajdev, C. Ellison, P.P. Irazoqui, Brain Res. 1282, 183 (2009).

4. J.J. Daly, J.R. Wolpaw, Lancet Neurol. 7, 1032 (2008)

5. J.P. Donoghue, Neuron 60, 511 (2008).

6. J. Leach, A.K.H. Achyuta, S.K. Murthy, Front. Neuroeng. 2 (2010).

7. C.T. Moritz, S.I. Perlmutter, E.E. Fetz, Nature 456, 639 (2008)

8. A.B. Schwartz, Annu. Rev. Neurosci. 27, 487 (2004).

9. A.B. Schwartz, X.T. Cui, D.J. Weber, D.W. Moran, Neuron 52, 205 (2006).

10. G. Buzsáki, Nat. Neurosci. 7, 446 (2004).

11. M.A. Nicolelis, Nature 409, 403 (2001).

12. G.C. McConnell, H.D. Rees, A.I. Levey, C. Gutekunst, R.E. Gross, R.V. Bellamkonda, J. Neural Eng. 6, 56003 (2009).

13. V.S. Polikov, M.L. Block, J.M. Fellous, J.S. Hong, W.M. Reichert, Biomaterials 27, 5368 (2006).

14. J.C. Williams, R.L. Rennaker, D.R. Kipke, Brain Res. 4, 303 (1999).

15. R. Biran, D.C. Martin, P.A. Tresco, Exp. Neurol. 195, 115 (2005)

16. V.S. Polikov, P.A. Tresco, W.M. Reichert, J. Neurosci. Methods 148, 1 (2005).

17. D.H. Szarowski, M.D. Andersen, S. Retterer, A.J. Spence, M. Isaacson, H.G. Craighead, J.N. Turner, W. Shain, Brain Res. 983, 23 (2003).

18. M.D. Johnson, O.E. Kao, D.R. Kipke, J. Neurosci. Methods 160, 276 (2007).

19. C.S. Bjornsson, S.J. Oh, Y.A. Al-Kofahi, Y.J. Lim, K.L. Smith, J.N. Turner, S. De, B. Roysam, W. Shain, S.J. Kim, J. Neural Eng. 3, 196 (2006).

20. A.A. Sharp, H.V. Panchawagh, A. Ortega, R. Artale, S. Richardson-Burns, D.S. Finch, K. Gall, R.L. Mahajan, D. Restrepo, J. Neural Eng. 3, L23 (2006).

21. W. He, R.V. Bellamkonda, in Indwelling Neural Implants: Strategies for Contending with the In Vivo Environment, W.M. Reichert, Ed. (CRC Press, Boca Raton, FL, 2008).

22. Y. Zhong, R.V. Bellamkonda, J. R. Soc. Interface 5, 957 (2008).

23. P.A. Tresco, B.D. Winslow, Crit. Rev. Biomed. Eng. 39, 29 (2011).

24. Y.-T. Kim, R.W. Hitchcock, M.J. Bridge, P.A. Tresco, Biomaterials 25, 2229 (2004)

25. H. Lee, R.V. Bellamkonda, W. Sun, M.E. Levenston, J. Neural Eng. 2, 81 (2005)

26. G.C. McConnell, T.M. Schneider, D.J. Owens, R.V. Bellamkonda, IEEE Trans. Biomed. Eng. 54, 1097 (2007)

27. M.A. Lebedev, M.A. Nicolelis, Trends Neurosci. 29, 536 (2006).

28. A. Gilletti, J. Muthuswamy, J. Neural Eng. 3, 189 (2006).

29. J. Subbaroyan, D.C. Martin, D.R. Kipke, J. Neural Eng. 2, 103 (2005).

30. R. Biran, D.C. Martin, P.A. Tresco, J. Biomed. Mater. Res. Part A 82, 169 (2007).

31. J.C. Williams, J.A. Hippensteel, J. Dilgen, W. Shain, D.R. Kipke, J. Neural Eng. 4, 410 (2007).

32. D.H. Kim, D.C. Martin, Biomaterials 27, 3031 (2006).

33. W. Shain, L. Spataro, J. Dilgen, K. Haverstick, S. Retterer, M. Isaacson, M. Saltzman, J.N. Turner, IEEE Trans. Neural Syst. Rehabil. Eng. 11, 186 (2003).

34. R. Wadhwa, C.F. Lagenaur, X.T. Cui, J. Controlled Release 110, 531 (2006).
35. Y. Zhong, R.V. Bellamkonda, Brain Res. 1148, 15 (2007).

36. L. Spataro, J. Dilgen, S. Retterer, A.J. Spence, M. Isaacson, J.N. Turner, W. Shain, Exp. Neurol. 194, 289 (2005)

37. R.L. Rennaker, J. Miller, H. Tang, D.A. Wilson, J. Neural Eng. 4, L1 (2007).

38. C. Caruso, C. Mohn, A.L. Karara, V. Rettori, H. Watanobe, H.B. Schioth, A. Seilicovich, M. Lasaga, Neuroendocrinology 79, 278 (2004).

39. W. He, G. McConnell, T. Schneider, R. Bellamkonda, Adv. Mater. 19, 3529 (2007).

40. P.J. Rousche, D.S. Pellinen, D.P. Pivin, J.C. Williams, R.J. Vetter, D.R. Kipke, IEEE Trans. Biomed. Eng. 48, 361 (2001).

41. J. Subbaroyan, D.R. Kipke, Conf. Proc. IEEE Eng. Med. Biol. Soc. 1, 3588 (2006).

42. S. Takeuchi, D. Ziegler, Y. Yoshida, K. Mabuchi, T. Suzuki, Lab Chip 5, 519 (2005).

43. B.A. Wester, R.H. Lee, M.C. LaPlaca, J. Neural Eng. 6, 024002 (2009).

44. L.J. Fernandez, A. Altuna, M. Tijero, G. Gabriel, R. Villa, M.J. Rodriguez, M. Batlle, R. Vilares, J. Berganzo, F.J. Blanco, J. Micromech. Microeng. 19, 025007 (2009)

45. A. Mercanzini, P. Colin, J.C. Bensadoun, A. Bertsch, P. Renaud, IEEE Trans. Biomed. Eng. 56, 1909 (2009).

46. Y. Lu, M.S. Shoichet, Biomaterials 30, 4143 (2009)

47. K. Lee, J. He, R. Clement, S. Massia, B. Kim, Biosens. Bioelectron. 20, 404 (2004).

48. S.A. Nikles, D.S. Pellinen, J. Kitagawa, R.M. Bradley, D.R. Kipke, K. Najafi, Engineering in Medicine and Biology Society, 2003. Proceedings of the 25th Annual International Conference of the IEEE, vol. 4, 3340 (2003).

49. S. Takeuchi, T. Suzuki, K. Mabuchi, H. Fujita, J. Micromech. Microeng. 14 104 (2004)

50. T.D. Kozai, D.R. Kipke, J. Neurosci. Methods 184, 2 (2009).

51. L. Kee-Keun, J. He, A. Singh, B. Kim, Proceedings of the International Conference on MEMS, NANO and Smart Systems 2003 (2003), p. 418.

52. L. Kee-Keun, J. He, A. Singh, S. Massia, G. Ehteshami, B. Kim, G. Raupp, J. Micromech. Microeng. 14, 32 (2004).

53. K. Potter, B. Gui, J.R. Capadona, in Biomimetics-Innovation Thru Mimicking Natures Inventions, Y. Bar-Cohen, Ed. (CRC Press, Boca Raton, FL, 2011), vol. 2, pp. 95-129.

54. Y.B. Lu, K. Franze, G. Seifert, C. Steinhauser, F. Kirchhoff, H. Wolburg, J. Guck, P. Janmey, E.Q. Wei, J. Kas, A. Reichenbach, Proc. Natl. Acad. Sci. U.S.A. 103, 17759 (2006)

55. J.P. Seymour, D.R. Kipke, Biomaterials 28, 3594 (2007)

56. K. Lee, A. Singh, J. He, S. Massia, Sens. Actuators, B 102, 67 (2004).

57. D. Lewitus, K.L. Smith, W. Shain, J. Kohn, Acta Biomater. 7, 2483 (2011).

58. K.A. Ludwig, J.D. Uram, J. Yang, D.C. Martin, D.R. Kipke, J. Neural Eng. 3, 59 (2006).

59. D.H. Kim, J.A. Wiler, D.J. Anderson, D.R. Kipke, D.C. Martin, ActaBiomater 6, 57 (2010).

60. X.T. Cui, D.C. Martin, Sens. Actuators, B 89, 92 (2003)

61. Y.H. Xiao, X. Cui, J.M. Hancock, M. Bouguettaya, J.R. Reynolds, D.C. Martin, Sens. Actuators, B 99, 437 (2004).

62. X.T. Cui, D.D. Zhou, IEEE Trans. Neural Syst. Rehabil. Eng. 15, 502 (2007).

63. Y.H. Xiao, D.C. Martin, X.T. Cui, M. Shenai, Appl. Biochem. Biotechnol. 128, $117(2006)$

64. D.H. Kim, M. Abidian, D.C. Martin, J. Biomed. Mater. Res. Part A 71, 577 (2004).

65. J. Yang, D.H. Kim, J.L. Hendricks, M. Leach, R. Northey, D.C. Martin, ActaBiomater. 1, 125 (2005).

66. X.T. Cui, J.F. Hetke, J.A. Wiler, A.J. Anderson, D.C. Martin, Sens. Actuators, A 93, 8 (2001).

67. X.T. Cui, V.A. Lee, Y. Raphael, J.A. Wiler, J.F. Hetke, D.J. Anderson, D.C. Martin, J. Biomed. Mater. Res. 56, 261 (2001).

68. J.R. Capadona, K. Shanmuganathan, D.J. Tyler, S.J. Rowan, C. Weder, Science 319, 1370 (2008).

69. J.R. Capadona, K. Shanmuganathan, S. Trittschuh, S. Seidel, S.J. Rowan, C. Weder, Biomacromolecules 10, 712 (2009).

70. J.R. Capadona, O. van den Berg, L.A. Capadona, M. Schroeter, S.J. Rowan, D.J. Tyler, C. Weder, Nat. Nano 2, 765 (2007).

71. K. Shanmuganathan, J.R. Capadona, S.J. Rowan, C. Weder, Prog. Polym. Sci. 35, 212 (2010).

72. K. Shanmuganathan, J.R. Capadona, S.J. Rowan, C. Weder, J. Mater. Chem. 20, $180(2010)$.

73. K. Shanmuganathan, J.R. Capadona, S.J. Rowan, C. Weder, ACS Appl. Mater. Interfaces 2, 165 (2009).

74. 0. van den Berg, J.R. Capadona, C. Weder, Biomacromolecules 8, 1353 (2007).

75. J.A. Trotter, T.J. Koob, J. Exp. Biol. 198, 1951 (1995)

76. T.J. Koob, M.M. Koob-Emunds, J.A. Trotter, J. Exp. Biol. 202, 2291 (1999). 
77. J.A. Trotter, J. Tipper, G. Lyons-Levy, K. Chino, A.H. Heuer, Z. Liu, M. Mrksich, C. Hodneland, W. Shannon-Dillmore, T.J. Koob, M.M. Koob-Emunds, K. Kadler, D. Holmes, Biochem. Soc. Trans. 28, 357 (2000).

78. T. Motokawa, Comput. Biochem. Physiol. 70, 41 (1981).

79. I.C. Wilkie, J. Exp. Biol. 205, 159 (2002).

80. I.C. Wilkie, Echinoderm Stud. 5, 61 (1996).

81. G.K.A.S. Szulgit, R.E. Shadwick, J. Exp. Biol. 203, 1539 (2000).

82. L. Hsu, S.J. Rowan, C. Weder, J. Mater. Chem. 21, 2812 (2011)

83. S.J. Eichhorn, A. Dufresne, M. Aranguren, J.R. Capadona, S.J. Rowan, C. Weder, W. Thielemans, M. Roman, S. Renneckar, W. Gindl, S. Weigel, H. Yano, K. Abe, M. Nogi, A. Mangalam, J. Simonsen, A.D. Benight, A. Bismarck, L.A. Berglund, J. Mater. Sci. 45, 1 (2010).

84. A. Sturcova, G.R. Davies, S.J. Eichorn, Biomacromolecules 6, 1055 (2005).

85. R. Rusli, S.J. Eichhorn, Appl. Phys. Lett. 93, 033111 (2008).

86. Y.C. Hsieh, H. Yano, M. Nogi, S.J. Eichhorn, Cellulose 15, 507 (2008).

87. M. Schroers, A. Kokil, C. Weder, J. Appl. Polym. Sci. 93, 2883 (2004).
88. M. Takayanagi, S. Uemura, S. Minami, J. Polym. Sci. C 5, 113 (1964) 89. N. Ouali, J.Y. Cavaillé, J. Pérez, Plast. Rubber Compos. Process. Appl. 16, 55 (1991).

90. R. Rusli, K. Shanmuganathan, S.J. Rowan, C. Weder, S.J. Eichhorn, Biomacromolecules 11, 762 (2010)

91. R. Rusli, K. Shanmuganathan, S.J. Rowan, C. Weder, S.J. Eichhorn, Biomacromolecules 12, 1363 (2011).

92. R. Rusli, S.J. Eichhorn, Appl. Phys. Lett. 93, 033111 (2008).

93. J. Mendez, P.K. Annamalai, S.J. Eichhorn, R. Rusli, S.J. Rowan, E.J. Foster, C. Weder, Macromolecules 44, 6827 (2011).

94. J.P. Harris, A.E. Hess, S.J. Rowan, C. Weder, C.A. Zorman, D.J. Tyler, J.R. Capadona, J. Neural Eng. 8, 046010 (2011).

95. A. Hess, J.R. Capadona, K. Shanmuganathan, L. Hsu, S.J. Rowan, C. Weder, D.J. Tyler, C.A. Zorman, J. Micromech. Microeng. 21, 54009 (2011).

96. J.P. Harris, J.R. Capadona, R.H. Miller, B.C. Healy, K. Shanmuganathan, S.J. Rowan, C. Weder, D.J. Tyler, J. Neural Eng. 8, 066011 (2011).

97. D.J. Loane, K.R. Byrnes, Neurotherapeutics 7, 366 (2010).

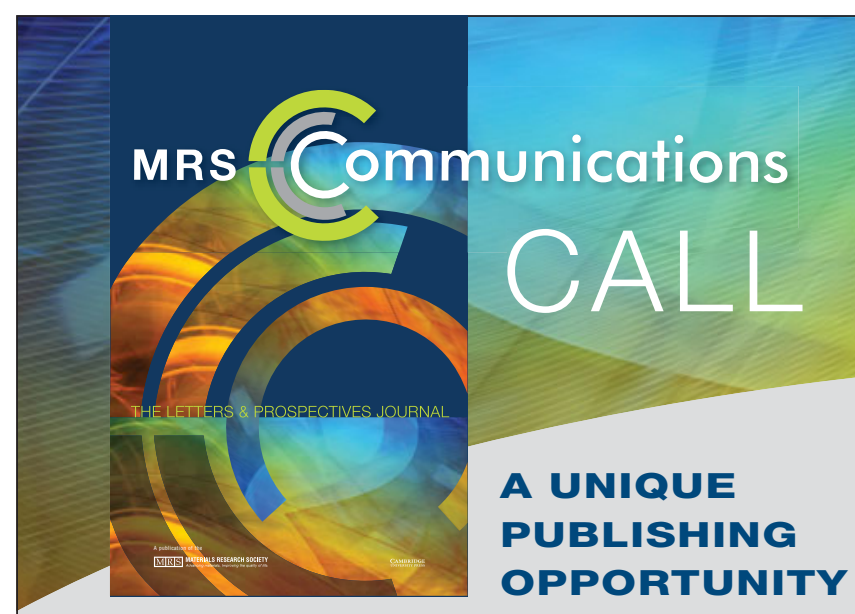

Manuscripts are being solicited for MRS Communications - a new full-color, high-impact journal focused on groundbreaking work across the broad spectrum of materials research.

Published jointly by the Materials Research Society (MRS) and Cambridge University Press, MRS Communications offers a rapid but rigorous peer-review process and time to publication. An aggressive production schedule will bring your article to online publication and a global audience within a target 14-day process from acceptance.

Hosted on the cutting-edge Cambridge Journals Online (CJO) platform, the journal features a robust suite of author and reader services, as well as an immediate reader/subscriber base including almost 16,000 MRS members and over 2,500 academic, industrial and government libraries worldwide.

Major article types for MRS Communications include:

\section{Research Letters}

Ultra-Rapid Communications

Prospectives Articles

Editorials

Commentaries

Correspondence

Prospectives Articles are a unique feature of this journal, offering succinct and forward-looking reviews of topics of interest to a broad materials research readership. For more information about the journal and/or these major article types, visit www.mrs.org/mrc or email mrc@mrs.org.
Manuscripts are solicited in the following topical areas, although submissions that succinctly describe groundbreaking work across the broad field of materials research are encouraged.

- Biomaterials and biomimetic materials

- Carbon-based materials

- Complex oxides and their interfaces

- Materials for energy storage, conversion and environmental remediation

- Materials for nanophotonics and plasmonic devices

- Theory and simulation of materials

- Mechanical behavior at the nanoscale

- Nanocrystal growth, structures and properties, including nanowires and nanotubes

- Nanoscale semiconductors for new electronic and photonic applications

- New materials synthesis, templating and assembly methods

- New topics in metals, alloys and transformations

- Novel and in-situ characterization methods

- Novel catalysts and sensor materials

- Organic and hybrid functional materials

- Quantum matter

- Surface, interface and length-scale effects on materials properties

For manuscript submission instructions, please visit www.mrs.org/mrc-instructions. 